\title{
EP-16
}

\section{Perioperative and oncologic outcomes of right anterior sectionectomy for liver disease: A single-center experience with 415 patients}

Jae Hoon LEE*, Jaewoo KWON, Yejong PARK, Woohyung LEE, Ki Byung SONG, Dae Wook HWANG, Song Cheol KIM, Young Joo LEE

Division of Hepato-Biliary and Pancreatic Surgery, Department of Surgery, Asan Medical Center, University of Ulsan College of Medicine, Seoul, Korea

Introduction: Right anterior sectionectomy (RAS) is technically difficult and performed infrequently, so there were a few reports of small numbers about this surgery. We described large number of clinicopathologic and oncologic outcomes associated with RAS

Methods: We retrospectively reviewed the medical records of 415 patients treated with RAS for hepatic tumors located at segment 5 and/or 8 between January 2008 and December 2017.

Results: All patients underwent RAS with the alternative Glissonean pedicle clamp and Kelly clamp-crushing methods for transection. The mean operative time was 165 minutes and the mean transection time was 28 minutes. Major morbidities ( $\geq$ grade III) occurred in 28 cases (6.7\%). Bile leakage occurred in 63 patients (15.1\%), but no patients required reoperation. Grade A/B/C post-hepatectomy liver failure occurred in 39/7/0 (9.4\%/1.7\%/0.0\%) patients, respectively. There were no in-hospital mortality caused by postoperative complications. The mean hospital stay was 13.3 days. The most common diagnosis was hepatocellular carcinoma $(\mathrm{HCC} ; \mathrm{n}=$ $361,87.0 \%)$, followed by intrahepatic cholangiocarcinoma $(n=15,3.6 \%)$, mixed HCC and cholangiocarcinoma $(n=17,4.1 \%)$, colorectal cancer liver metastasis $(\mathrm{n}=12,2.9 \%)$. The mean tumor size was $3.8 \mathrm{~cm}$. Among HCC patients, the 5- and 10-year overall survival (OS) rate was 78.3\%, 64.4\%, and 5- and 10- year disease-free survival (DFS) rate was 57.2\%, 37.7\%, respectively. Operative time, tumor size, and vessel invasion were factors significantly associated with OS and DFS for HCC patients.

Conclusions: RAS was associated with acceptable procedure-related morbidity and mortality as well as appropriate oncologic outcomes for HCC patients. 